\title{
Retraction Note to: Systemische Resilienz - Die Perspektiven und das Resilienz-Quadarat
}

\section{Günther Josef Mohr}

Online publiziert: 27. Januar 2021

(C) Springer Fachmedien Wiesbaden GmbH, ein Teil von Springer Nature 2021

\section{Retraction Note to:}

https://doi.org/10.1007/s11613-016-0476-y

Dieser Artikel von Günther Josef Mohr (2016) wurde von der Herausgeberin zurückgezogen, da er signifikant wörtliche Übereinstimmungen mit der Dissertation von Jessica Di Bella (2014) beinhaltet, die nicht entsprechend zitiert wurden.

Der Autor Günther Josef Mohr stimmt dieser Retraction zu.

The editor has retracted this article by Günther Josef Mohr (2016) because of significant overlap with the dissertation of Jessica Di Bella (2014) without proper citation.

The author Günther Josef Mohr agrees to this retraction.

\section{Literatur}

Di Bella, J. (2014). Unternehmerische Resilienz. Protektive Faktoren für unternehmerischen Erfolg in risikoreichen Kontexten. Dissertation. Mannheim: Universität Mannheim.

Mohr, G. J. (2016). Systemische Resilienz - Die Perspektiven und das Resilienz-Quadarat. Organisationsberat Superv Coach, 23, 411-426. https://doi.org/10.1007/s11613-016-0476-y.

Die Online-Version des Originalartikels ist unter https://doi.org/10.1007/s11613-016-0476-y zu finden.

Dipl.-Psych./Dipl.-Volksw. G. J. Mohr ( $\triangle)$

Klarastr. 7, 65719 Hofheim, Deutschland

E-Mail: info@mohr-coaching.de 